\title{
Study of Poly(ethylene oxide) Electrolyte with Polyurethane/Sulfonate Side Chains
}

\author{
XINLING WANG, ${ }^{1}$ BIN FANG, ${ }^{1}$ LEI WANG, ${ }^{1}$ XIAOZHEN TANG, ${ }^{1}$ FENG-CHIH CHANG ${ }^{2}$ \\ ${ }^{1}$ School of Chemistry and Chemical Technology, Shanghai Jiaotong University, Shanghai 200030, China \\ ${ }^{2}$ Department of Applied Chemistry, National Chiao-Tung University, Hsin-Chu, Taiwan
}

Received 1 April 2000; accepted 24 December 2000

\begin{abstract}
Sulfonated dimethyl fumarate (SDMF) was prepared. Poly(ethylene oxide) (PEO) with sodium sulfonate side chains (SPEO) was synthesized by transesterification between SDMF and PEO with molecular weights of 200, 400, 600, 800, and 1000. The SPEO was subsequently mixed as a plasticizer with PEO polyurethane (PU). Samples were characterized by elemental analysis, FTIR, ${ }^{1} \mathrm{H}-\mathrm{NMR}$, gel permeation chromatography, and impedance analysis. The mixture exhibited a homogeneous domain. The maximum conductivity of the CPU1000/SPEO600 was $2.6 \times 10^{-7} \mathrm{~S} \mathrm{~cm}^{-1}$ at room temperature. The relation between the ionic conductivity and the temperature was in agreement with the Arrhenius equation. () 2001 John Wiley \& Sons, Inc. J Appl Polym Sci 82: 541-545, 2001
\end{abstract}

Key words: polyurethane; blend; polymer electrolyte

\section{INTRODUCTION}

Single ionic conductive polymer electrolytes have attracted growing interest because of their lower degree of polarization. ${ }^{1-3}$ These electrolytes display a high ionic conductivity of about $10^{-6}-10^{-8}$ $\mathrm{S} \mathrm{cm}{ }^{-1}$ at room temperature. The hard or soft segment polyurethanes (PUs) are used as single ionic conductor polymer electrolytes because of their good balance of chemical stability and mechanical properties. In previous study a new kind of single ionic polymer electrolyte called soft segment ionic PU was synthesized, and these materials possessed ambient conductivities of about $10^{-6}-10^{-7} \mathrm{~S} \mathrm{~cm}^{-1}{ }^{4,5}$ The synthesis and purification of these hard or soft segment ionic PUs was very difficult. ${ }^{4,5}$

Correspondence to: X. Wang (shenxw@online.sh.cn).

Journal of Applied Polymer Science, Vol. 82, 541-545 (2001)

(C) 2001 John Wiley \& Sons, Inc.
In this article we tried to find a simpler method for the preparation of the single ionic PU electrolytes.

\section{EXPERIMENTAL}

\section{Materials}

Poly(ethylene oxide)s (PEO) with molecular weights of 200,400,600, 800, and 1000 (Aldrich) were dried under a vacuum at $60^{\circ} \mathrm{C}$ for $48 \mathrm{~h}$ before use. The 4,4'-methylenebis(phenyl isocyanate) (MDI, Aldrich) was distilled under a vacuum and used immediately. The $N, N$-dimethylacetamide (DMA) and 1,4-butanediol (BD) from Riedel-de Haën were dried by refluxing over calcium hydride $\left(\mathrm{CaH}_{2}\right.$, Aldrich) for $4 \mathrm{~h}$ to exclude trace water and then distilled under a vacuum. Sodium hydrogen sulfite $\left(\mathrm{NaHSO}_{3}\right)$, dimethyl sulfoxide (DMSO), and calcium hydride were used without further purification. 


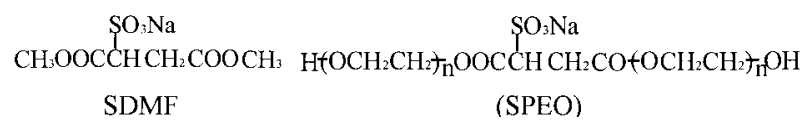

Scheme 1 The chemical structures of SDMF and SPEO.

\section{Sample Preparation}

Sulfonated dimethyl fumarate (SDMF) was prepared by dissolving $\mathrm{DMF}$ and $\mathrm{NaHSO}_{3}$ in a methanol and water solution and refluxing at $80^{\circ} \mathrm{C}$ for $8 \mathrm{~h}$. Sodium sulfonate side chain PEO (SPEO) was prepared by mixing SDMF, PEO, and a catalyst under a nitrogen atmosphere at $175^{\circ} \mathrm{C}$ for $5 \mathrm{~h}$. The purification of SDMF and SPEO was reported in a previous article. ${ }^{4}$ Their chemical structures are shown in Scheme 1.

In preparing the PEO PU or crosslinked PEO PU (CPU) the DMA solution containing PEO was added dropwise to the DMA solution containing MDI under a nitrogen atmosphere at $40^{\circ} \mathrm{C}$ for $3 \mathrm{~h}$. Subsequently, the DMA solution containing BD to prepare the PU or the mixture of $\mathrm{BD}$ and glycerol to prepare the CPU (molar ratio of $\mathrm{BD}$ :glycerol $>10: 1$ ) was added dropwise over a period of 30 min and continually reacted for $1.5 \mathrm{~h}$. The degree of crosslinking of the CPU was low. The polymer formed was precipitated and recovered in a large amount of distilled water. The polymer was filtered and dried under a vacuum at $60^{\circ} \mathrm{C}$ for $24 \mathrm{~h}$.
To prepare the polymer electrolytes the PU or CPU and SPEO were first dissolved in DMF (5 wt $\%$ ), and cast on a Teflon plate. After the solvent was evaporated at $60-70^{\circ} \mathrm{C}$ the films were transferred to a vacuum oven and dried at $60^{\circ} \mathrm{C}$ for at least $48 \mathrm{~h}$ to remove the residual solvent.

\section{Measurement}

Ionic conductivity measurements with alternating current were carried out with an electrochemical impedance system in a frequency range of $10-10^{6} \mathrm{~Hz}$. The IR spectra were recorded on a Nicolet FTIR-502 at a resolution of $2 \mathrm{~cm}^{-1}$. The thermograms of the PU or polymer electrolytes were recorded using a differential scanning calorimeter (DSC) at a heating rate of $10^{\circ} \mathrm{C} / \mathrm{min}$ from -120 to $150^{\circ} \mathrm{C}$.

\section{RESULTS AND DISCUSSION}

The SDMF and SPEOs were characterized by ${ }^{1} \mathrm{H}$ NMR spectra, elemental analyses, and gel permeation chromatography. The results indicated that the SDMF and SPEO were consistent with the expected structures. ${ }^{4}$

The IR spectra (Fig. 1) of the PU/SPEO showed the following characteristic bands: $\mathrm{S}-\mathrm{O}$ stretching at 1039 and $1163 \mathrm{~cm}^{-1}$ and $\mathrm{C}-\mathrm{O}-\mathrm{C}$ stretching at 1100 and $1222 \mathrm{~cm}^{-1}$.

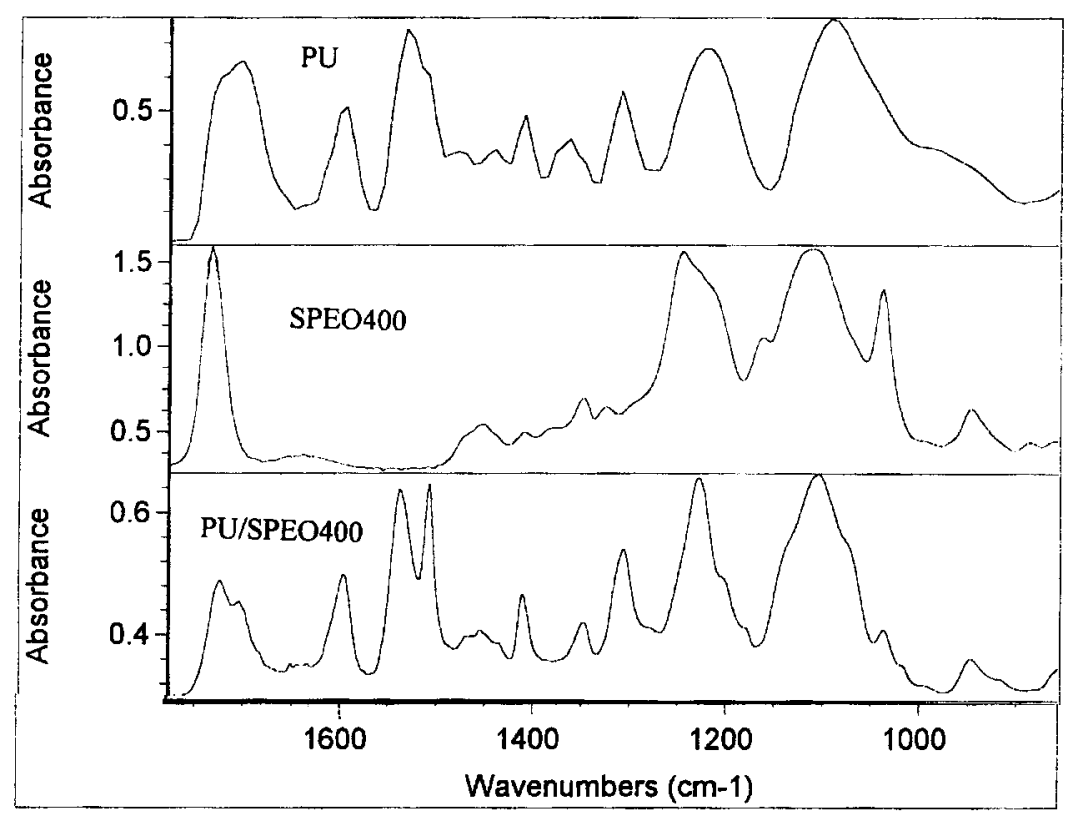

Figure 1 IR Spectra of PU, SPEO400, and PU/SPEO400. 


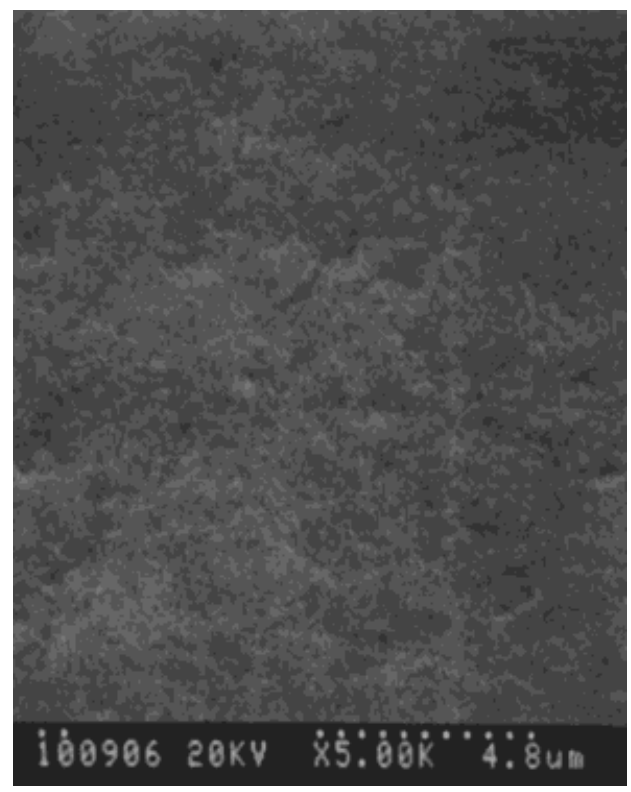

Figure 2 A scanning electron micrograph of CPU1000/SPEO1000.

A typical scanning electron micrograph of the surface of the CPU1000/SPEO is shown in Figure 2 . Figure 2 indicates that the morphology of the CPU1000/SPEO was homogenous. It is possible that the sodium ions in the SPEO were able to improve their compatibility.

The DSC measurement results of CPU1000/ SPEO are shown in Figure 3. The DSC data and the physical data for the CPU/SPEO are listed in
Table I DSC Measurement Results and Physical Data of CPU/SPEO

\begin{tabular}{lrrrr}
\hline \multicolumn{1}{c}{ Sample } & $\begin{array}{c}T_{g} \\
(\mathrm{~K})\end{array}$ & $\begin{array}{c}\Delta T_{g} \\
(\mathrm{~K})^{*}\end{array}$ & {$[\mathrm{E} 0] /[\mathrm{Na}]$} & $\begin{array}{c}\mathrm{Na}^{+} \\
(\%)\end{array}$ \\
\hline CPU1000 & 245.5 & - & - & - \\
CPU1000/SPEO200 & 240.4 & 5.1 & $44 / 1$ & 0.98 \\
CPU1000/SPEO400 & 235.7 & 9.8 & $51 / 1$ & 0.57 \\
CPU1000/SPEO600 & 232.2 & 13.3 & $74 / 1$ & 0.42 \\
CPU1000/SPEO800 & 233.1 & 12.4 & $100 / 1$ & 0.32 \\
CPU1000/SPEO1000 & 238.0 & 7.5 & $120 / 1$ & 0.26 \\
\hline
\end{tabular}

$\Delta T_{g}$, the difference in the glass-transition temperatures $\left(T_{g}\right)$ of CPU1000 and CPU1000/SPEO.

Table I. Figure 4 indicates that the glass-transition temperature $\left(T_{g}\right)$ of the mixture decreased with the increase of the PEO molecular weight from 200 to 600 . There was little effect on the $T_{g}$ of the mixture with the increase of the PEO molecular weight from 600 to 1000 . The crosslinking of the PU was suitable to reduce the $T_{g}$ of the polymer electrolyte. The $T_{g}$ of CPU1000 was lower than that of CPU900. It is possible that the molecular chains of PEO1000 in the CPU were more flexible. The effect of the PEO molecular weight in the SPEO on the conductivity of the mixture is shown in Figure 5. The conductivity of the mixture was increased with the increase of the PEO molecular weight from 200 to 600 , but there was little affect with the increase of the PEO molecular weight from 600 to 1000 . The

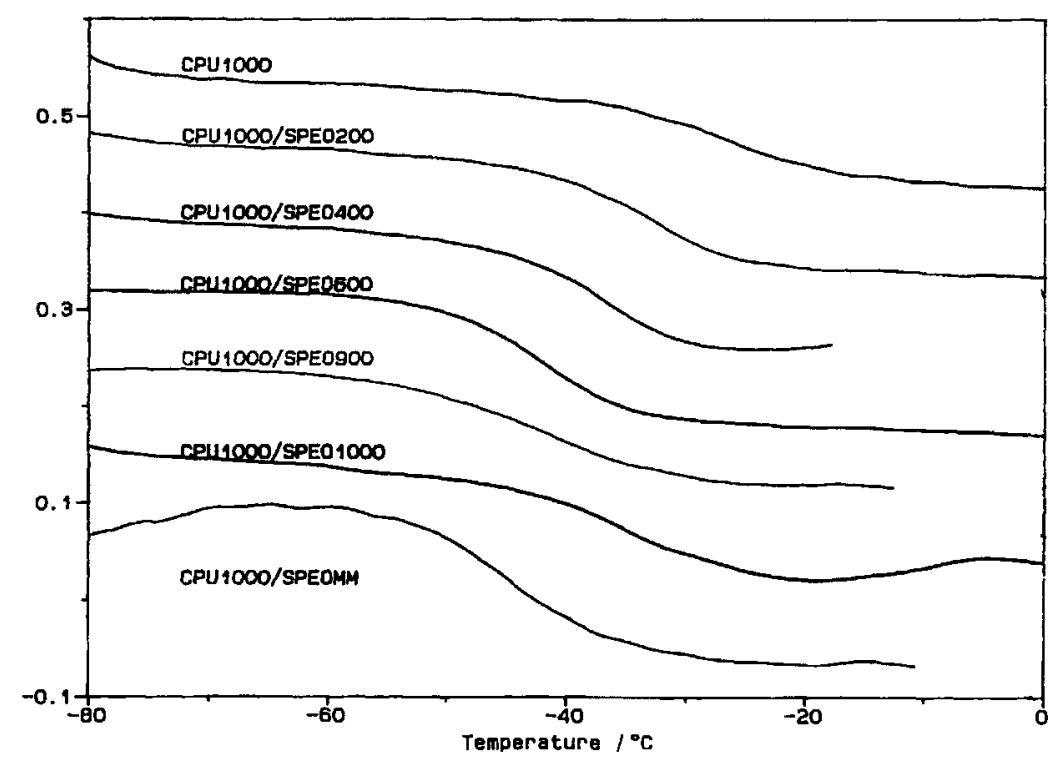

Figure 3 The DSC measurement results of CPU1000/SPEO. 


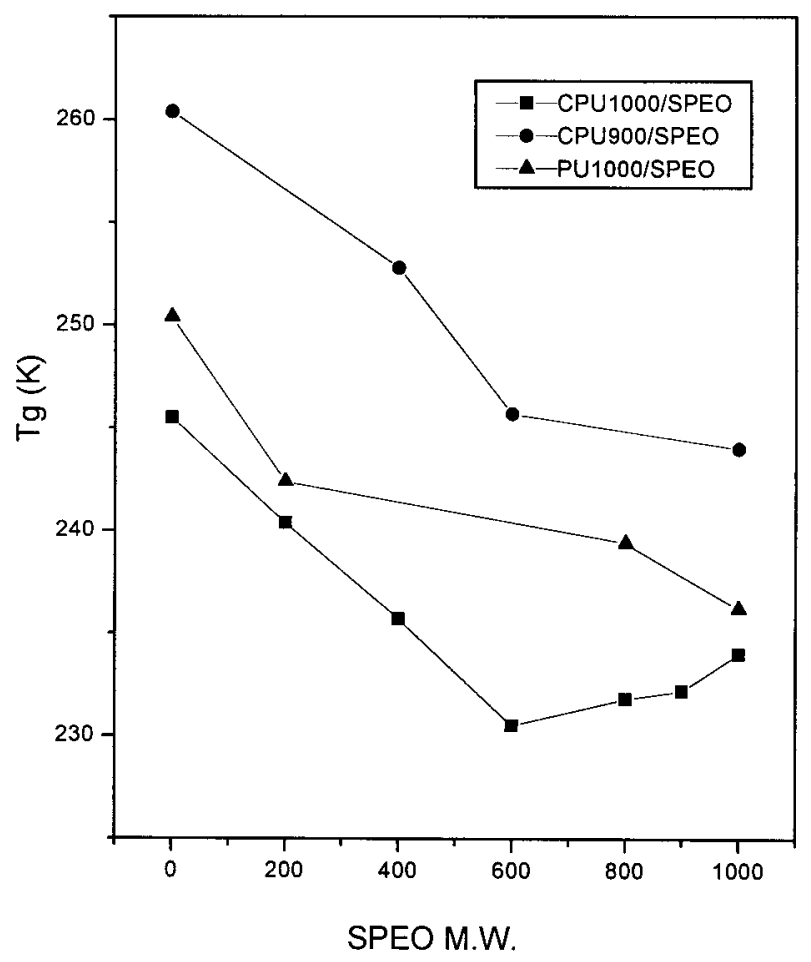

Figure 4 The effect of the PEO molecular weight (MW) in SPEO on the glass-transition temperature $\left(T_{g}\right)$ of the mixture.

results of the ionic conductivity and the $T_{g}$ of the polymer electrolyte were in agreement. The conductivity of CPU1000/SPEO was the highest. In the PU/SPEO or CPU/SPEO systems the $T_{g}$ was influenced by two contrary facts: the plasticizing effect and the sodium ionic concentration. The plasticizing effect was decreased in the polymer electrolyte with the increase of the SPEO molecular weight because the molar ratio of SPEO decreased and the length of the polymer chain increased, resulting in the $T_{g}$ increase of the mixture. On the other hand, the sodium ionic concentration decreased with increased PEO molecular weight in the SPEO and resulted in a decreased $T_{g}$ of the mixture. The conductivity of the polymer electrolyte was facilitated by the segmental mobility of the polymer chains. This suggested that the polymer electrolyte would exhibit higher ionic conductivity with the decrease of its $T_{g}$. The conductivity of CPU1000/SPEO600 was $2.6 \times 10^{-7} \mathrm{~S} \mathrm{~cm}^{-1}$ at room temperature.

Figure 6 shows the temperature dependence of the ionic conductivity of CPU1000/SPEO. The ionic conductivity of CPU1000/SPEO was increased by the increase of the temperature. The

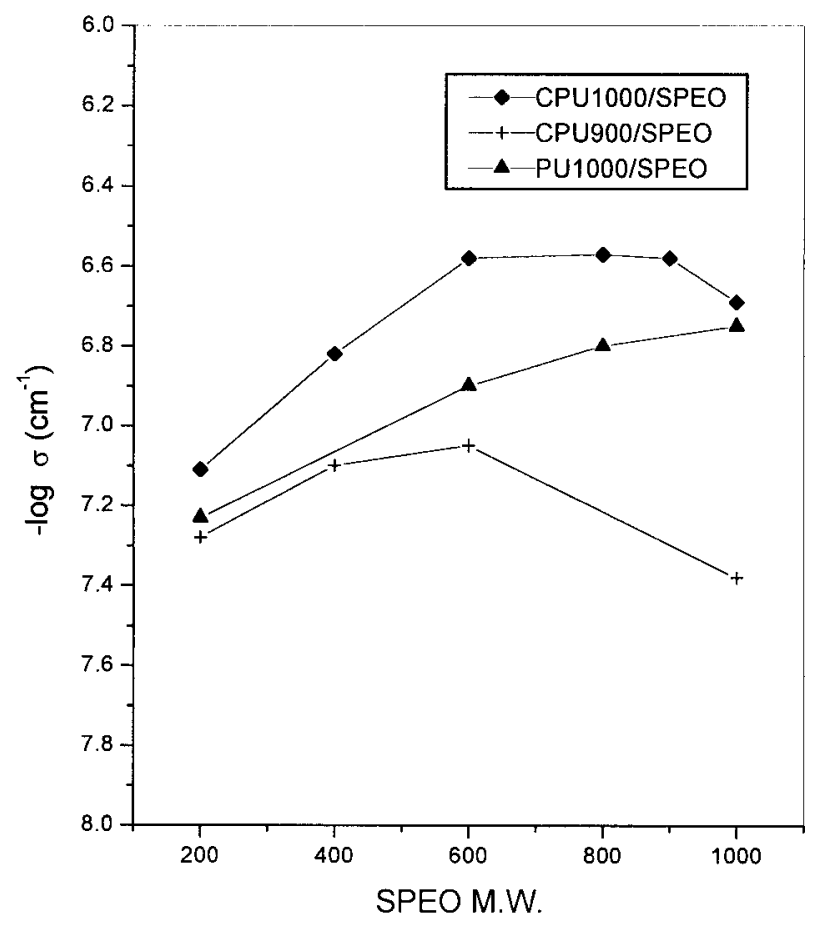

Figure 5 The effect of the PEO molecular weight (MW) in SPEO on the conductivity $(\sigma)$ of the mixture.

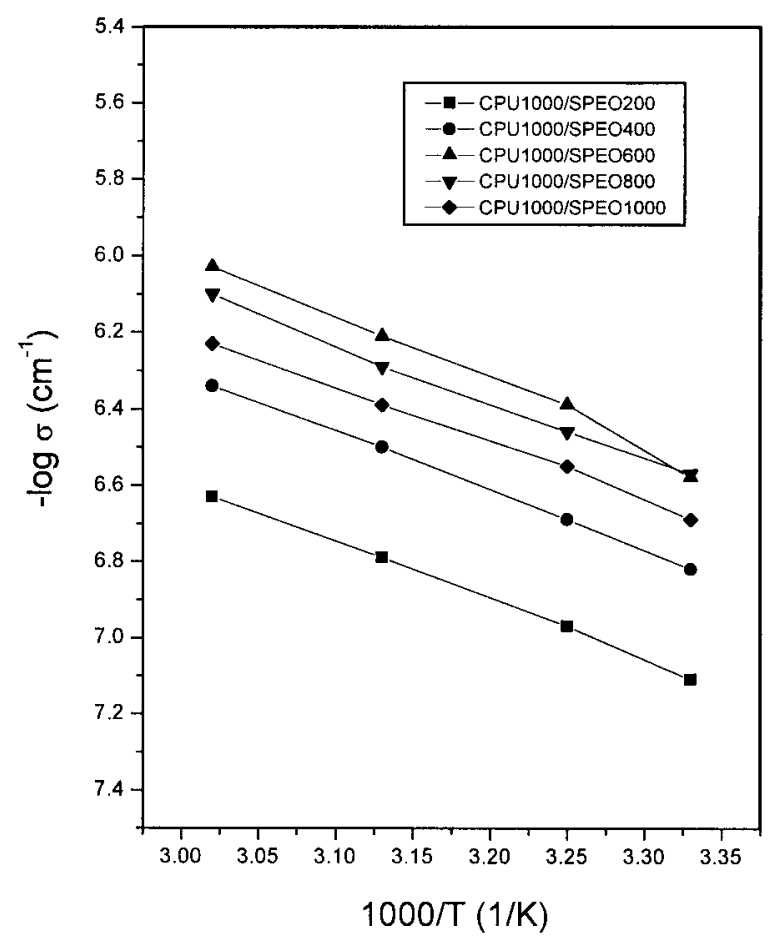

Figure 6 Arrhenius plots of CPU1000/SPEO. 
relation between the ionic conductivity and the temperature was in agreement with the Arrhenius equation.

\section{CONCLUSIONS}

Mixing PU and SPEO is a convenient method to attain a single ionic polymer electrolyte. The conductivity of the mixture was the highest when the molecular weight of PEO was 600 and 800 . We found that crosslinking of the PU caused improvement of the film dimensional stability and enhanced the ionic conductivity of the blend. The conductivity of CPU1000/SPEO600 was 2.7 $\times 10^{-7} \mathrm{~S} \mathrm{~cm}^{-1}$ at room temperature when the
$[\mathrm{EO}] /[\mathrm{Na}]$ was $74 / 1$. The relation between the ionic conductivity and the temperature was in agreement with the Arrhenius equation.

\section{REFERENCES}

1. Hardy, L. C.; Shriver, D. F. J Am Chem Soc 1985, $107,3823$.

2. Benrabah, D.; Sylla, S.; Alloin, F. Electrochim Acta 1995, 40, 2259.

3. Fujinami, T.; Tokimnne, A.; Anne Metta, M. Chem Mater 1997, 9, 2236.

4. Wang, X. L.; Li, H.; Tang, X. Z.; Chang, F. Z. J Polym Sci Part B 1999, 37, 837.

5. Wang, L.; Yang, B.; Wang, X. L. J Appl Polym Sci 1999, 71, 1711. 